\title{
Quantum Mechanical Simulation of Vibration-torsion-rotation Levels of Methanol
}

\author{
Yun-Bo Duan ${ }^{1}$ and Anne B. McCoy ${ }^{2}$ \\ ${ }^{1}$ Institute for Computational Science and Engineering, \\ Ocean University of Qingdao, Shandong 266003, China \\ and \\ Department of Physics and Astronomy, \\ The University of British Columbia, \\ Vancouver, BC V6T 1Z1, Canada \\ yduan@physics.ubc.ca \\ ${ }^{2}$ Department of Chemistry, The Ohio State University, \\ Columbus, OH 43210 \\ mccoy@chemistry.ohio-state.edu
}

\begin{abstract}
Two kinds of vibration-torsion-rotation Hamiltonians, referred as a model Hamiltonian and quantum mechanical Hamiltonian, are constructed to investigate the vibration-torsion-rotational interaction in methanol. The model Hamiltonian is based on the formulation of reduction of Hamiltonian in which the CO-stretching mode $\nu_{8}$, the large-amplitude torsion mode $\nu_{12}$ and the three degrees of freedom that correspond to the overall rotation of the molecule are considered simultaneously. This Hamiltonian is used to carry out an analysis of already published data for $\mathrm{CH}_{3} \mathrm{OH}$ with $v_{c o} \leq 1$ (CO-stretching vibrational quantum number), $v_{t} \leq 4$ (torsional quantum number), and $J \leq 5$. The relative locations of the CO-stretch vibrational ground state and the fundamental state are well reproduced for torsional states with $v_{t} \leq 4$ and $J \leq 5$. An effective potential energy surface that describes $\nu_{8}$ and $\nu_{12}$ modes is obtained from this analysis. The quantum mechanical Hamiltonian is partitioned in the form $H_{A}+H_{B}+H_{\text {int }}$ by employing a body-fixed axis system and the Jacobi coordinates, where $H_{A}$ and $H_{B}$ are the rovibrational Hamiltonians of methyl group $\mathrm{CH}_{3}$ and asymmetric rotor $\mathrm{OH}$, and $H_{\text {int }}$ represents their interactions. This Hamiltonian is used to carry out a pure quantum mechanical simulation of the CO-stretching-torsion-rotational states usingthe the potential function obtained from the model Hamiltonian analysis. The present analyses show that a variety of resonance interactions can affect states for energies larger than $1100 \mathrm{~cm}^{-1}$.
\end{abstract}

V.N. Alexandrov et al. (Eds.): ICCS 2001, LNCS 2074, pp. 893-902, 2001.

(C) Springer-Verlag Berlin Heidelberg 2001 


\section{Introduction}

The interactions between large-amplitude internal motions, overall rotation and other vibrational motions in methanol that contains a three-fold internal rotational potential barrier lead to the complicated energy level patterns. ${ }^{1,2}$ The investigation of vibration-torsion-rotation (VTR) effects in molecules with largeamplitude internal motions has assumed increasing prominence as modern experimental and computational technologies have progressed such that obtaining gas-phase spectra of a wide variety of internal-rotation-type molecules with very high resolution and thereby a full quantum mechanical description of the finer details associated with higher-order VTR effects through computer simulation are now possible. Indeed, these technologies have recently allowed great advances in understanding the observed spectra with high resolution in laboratory and interstellar space, such that many VTR effects and potential energy surfaces (PES) are now obtainable by inversion of experimental data or direct ab initio quantum chemical calculation. Paralleling these developments, many researchers have attempted to extend this success to investigation of Hamiltonian models and construction of more general potential energy surfaces, which are ultimately necessary to understand the properties of the molecular spectra, energy levels and transition intensities. Due to the extremely complicated energy level structure existing in the larger-amplitude internal-motion type molecules, for example methanol, the study of structure, dynamics and spectroscopy generally demands very accurate experimental and theoretical methods ${ }^{3-10}$.

To date, most of theoretical treatments of this type molecule have been based on Hamiltonians that are obtained using the one-large-amplitude internal rotation model. These treatments are based on the results of applying low order perturbation theory, through contact transformation, to the Hamiltonian, written in terms of either the normal or local mode coordinates. As a result of this process, a model Hamiltonian is parameterized in terms of a set spectroscopic constants by fitting observed spectrum. Generally, the model Hamiltonians based on one-large-amplitude internal rotation model have met with a high degree of success, for both symmetric and asymmetric internal rotors, in determining the intramolecular potential energy hindering the internal rotation. The most advantage of these models is that they can be applied to highly excited states of larger polyatomic molecules. However, The models have their drawback. First, for a simple model of a rigid rotor with a rigid internal rotor, many effects observable in the spectra of the molecule depend on the interaction of both internal rotation and overall rotation with the other vibrations. These effects should be considered with analyses. Second, the perturbation theory is based on the usual normal mode coordinates and is assumed to provide an exact description of the energy level progressions only for non-degenerate case. All of these assumption are not entirely valid. Third, a correlation-free form of Hamiltonian obtained using contact transformation method is different for different molecules and even different isotopomers of the same molecule. ${ }^{5}$ This indicates that the contact transformation procedure depends on the particular molecule under consideration. Finally, so many parameters in a practical application had 
to be used to achieve experimental precision that the physical picture of models used and the physical origin of the spectroscopic parameters fitted become ambiguous. ${ }^{8-10}$ Evidently, the usual normal mode expansion of the Hamiltonian are no longer valid when the system of interest contains one or more large amplitude vibrational motions that are highly anharmonic. The usual perturbation expansions for the effects of anharmonicity and VTR coupling may not converge.

For methanol, in addition to the low-frequency, large amplitude torsion, eleven other vibrational degrees of freedom are needed to describe the dynamics of the molecule. Difficulties in locating the fundamentals arise from the overlap of bands due to the broad rotational envelopes, from complicated torsional structure, and from Coriolis couplings and resonances. Thus, how to provide a realistic model system in which interactions among rotation, torsion and other vibrational modes are clearly considered is an important challenge. As the fundamental frequency of the CO-stretch mode is the nearest one to that of the torsional mode in methanol, the understanding of interactions between the CO-stretching mode and the large amplitude hindered internal rotation is an intrinsic fundamental problem. In fact, the CO-stretch fundamental has been studied extensively because of interest in the rich optically pumped far infrared laser emission observed from methanol. The problem encountered now is the observed CO-stretch torsion-rotation energy structure is still not properly accounted for by existing models even though $\nu_{8}$ is the second lowest frequency mode. In particular, the fact that the hindering barrier for torsional mode had to be increased by about $20 \mathrm{~cm}^{-1}$ relative to its value in the ground state indicates the presence of a significant torsion-CO-stretch interaction. Therefore, investigation of interaction between the CO-stretch fundamental and the large amplitude torsion is of considerable interest.

First, in the present work, we present a model Hamiltonian that accounts for the couplings among vibration, torsion and rotation for a molecule with an internal rotor. A five-dimensional VTR Hamiltonian for methanol is constructed based on the formulation of reduction of Hamiltonian in which the CO-stretching mode $\nu_{8}$, the large-amplitude torsion mode $\nu_{12}$ and the three degrees of freedom that correspond to the overall rotation of the molecule are considered simultaneously. This Hamiltonian is used to carry out an analysis of already published data for $\mathrm{CH}_{3} \mathrm{OH}$ with $v_{c o} \leq 1, v_{t} \leq 4, J \leq 5$, and $|K| \leq 5$. The present analysis indicates that the height of the torsional barrier $V_{3}$ becomes $370 \mathrm{~cm}^{-1}$ by introducing torsion-CO-stretching-vibration interaction terms, this should be compared to the values for $V_{3}$ when only $v_{c o}=0$ or $v_{c o}=1$ are considered $373 \mathrm{~cm}^{-1}$ for ground vibrational state and $392 \mathrm{~cm}^{-1}$ for the CO-stretching fundamental state. Second, instead of taking the perturbation approach for investigating the structure, dynamics and spectroscopy of methanol, we focus on the variational approach that based on an internal coordinate Hamiltonian and underlying potential function. The variational approach is a perturbationtheory-free method ${ }^{11}$ and it is possible to develop theoretical models applicable to all molecules in a certain class. This method has been extensively applied for weakly bond cluster molecules and chemical reaction dynamics. Employing a 
body-fixed axis system and the Jacobi coordinates, we derive a Hamiltonian for the vibrating-internally rotating-rotating $\mathrm{CH}_{3} \mathrm{OH}$ molecule with a large amplitude internal motion-vibration-rotation interactions. The resulting Hamiltonian is used to carry out a pure quantum mechanical simulation for the VTR states of this kind of molecule.

\section{Theory and Method}

\subsection{Perturbation Method - Model Hamiltonian}

In the present model, we consider simultaneously the small amplitude COstretching mode and the torsion with the overall rotation in methanol. The five degree-of-freedom model arises by freezing the other vibrational modes except the two modes with lowest frequency. The distance between the centers of mass of methyl group $\mathrm{CH}_{3}$ and asymmetric rotor $\mathrm{OH}, R$, is used to describe the CO-stretching vibration. A frame-fixed axis system with its origin at the center of mass of $\mathrm{CH}_{3} \mathrm{OH}$ is chosen in which the $a$ axis is parallel to the axis of symmetry of the internal rotor, the $b$ axis is parallel to the plane of symmetry, and the $c$ axis is perpendicular to the plane of symmetry. The internal rotation coordinate $\gamma$ is chosen as the torsional angle of the internal rotor with respect to the frame.

According to the perturbation theory, the reduced rovibrational Hamiltonian of methanol for a given total angular momentum $J$ can be written as

$$
H=H_{V}+H_{T}+H_{R}+H_{T R}+H_{V T R},
$$

where $H_{V}$ is the CO-stretching vibrational Hamiltonian, $H_{T}$ is the torsional Hamiltonian, $H_{R}$ is the overall rotation Hamiltonian, $H_{T R}$ is the reduced torsionrotational interaction Hamiltonian that is analogous to those described in Refs. 5 and 6 and is totally symmetric in the molecular symmetry (MS) group $G_{6}$ and invariant under time reversal, and $H_{V T R}$ represents interactions among vibration, torsion, and rotation, which is obtained by multiplying each of the terms in $H_{R}, H_{T}$ and $H_{T R}$ and a power series in $z$ and $P_{z}$.

The matrix elements of the complete CO-stretching-torsion-rotational Hamiltonian will be obtained by using the basis functions

$$
\left|\Psi_{v_{c o}, v_{t}, \sigma, J, K}\right\rangle=\left|v_{c o}\right\rangle\left|v_{t}, \sigma\right\rangle|J K\rangle,
$$

where the torsional symmetry label $\sigma$ is equal to $0,+1$, or -1 , classifying the levels respectively as $A, E_{1}$, or $E_{2}$ symmetry species of MS $G_{6}$ group associated with the threefold nature of the hindering potential. The VTR energy levels are obtained by diagonalizing the matrix resulting from the Hamiltonian matrix.

\subsection{Perturbation-free Method - Variational Calculation}

In the present model, methanol is divided into two subsystems $A$ and $B$, i.e., methyl group $\mathrm{CH}_{3}$ and asymmetric diatomic $\mathrm{OH}$. The five Jacobi vectors $\left(\mathbf{r}_{1 A}, \mathbf{r}_{2 A}\right.$, 
$\left.\mathbf{r}_{3 A}, \mathbf{r}_{B}, \mathbf{R}\right)$ specify the atomic positions in methanol, $\mathrm{CH}_{3} \mathrm{OH}$. To formulate our theory, three kinds of axis systems are introduced as follows: i) the space fixes (SF) axis system; ii) the body-fixed (BF) axis system $(x, y, z)$ with its origin at the molecular center of mass; iii) the monomer fixed (MF) axis system $(\xi, \eta, \zeta)$ with its origin at the nuclear center of mass of the monomer. The $\zeta$-axis is along with one of the instantaneous principal axes.

The coordinates used in the present work are defined as follows. In the BF coordinate frame, the $z$-axis is defined to lie along the Jacobi vector $\mathbf{R}$ that connects the centers of mass of the two fragments $A$ and $B$, pointing from the monomer $A$ to the monomer $B$. The symmetric plane, i,e., HCOH plane, of methanol coincides with the $x z$-plane with the $x$-axis points upward. This is the geometry with torsional angle setting to be zero. The $\mathbf{R}$ has the polar angles $(\alpha, \beta)$ with respect to the SF coordinate system. The MF frame of monomer $A$ as follows, (i) the $\zeta$-axis lies parallel to $\mathbf{r}_{3 A}$; (ii) $\mathbf{r}_{2 A}$ lies in the $\xi \zeta$-plane. Thus, the six independent coordinates that describe the internal vibrations in $\mathrm{CH}_{3}$ group are $\left(r_{1 A}, r_{2 A}, r_{3 A}, \theta_{1}, \phi_{1}, \theta_{2}\right)$. The Euler angles $\boldsymbol{\Omega}^{(\alpha)}=\left(\theta_{\alpha}, \phi_{\alpha}, \chi_{\alpha}\right)$ with $\alpha=A$ and $B$ describe the orientation of the MF axis systems of the methyl group $\mathrm{CH}_{3}$ and the $\mathrm{OH}$ group in the body fixed axes, where the dihedral angle $\phi=\phi_{B}-\phi_{A}$ describes the torsion of the fragments $A$ and $B$ about $z$-axis, $\chi_{A}$ describes rotation of $\mathrm{CH}_{3}$ about its symmetric axis. The angle $\chi_{B}$ and the corresponding quantum number disappear for methanol due to a linear part, $\mathrm{OH}$.

The full-dimensional rovibrational Hamiltonian of a molecule that can be divided into two monomers for a given total angular momentum $J$, in the BF axis system and the Jacobi coordinates above, can be written as

$$
H=H_{r v}^{(A)}+H_{r v}^{(B)}+H_{i n t}
$$

where $H_{r v}^{(\alpha)}$, with $\alpha=A$ and $B$, represents the rovibrational kinetic operators of the $\mathrm{CH}_{3}$ and $\mathrm{OH}$, respectively. The $H_{r v}^{(A)}$ can be written as

$$
H_{r v}^{(A)}=\sum_{i=1}^{2}\left[-\frac{\hbar^{2}}{2 \mu_{i A}} \frac{\partial^{2}}{\partial r_{i A}^{2}}+\frac{L_{i A}^{2}}{2 \mu_{i A} r_{i A}^{2}}\right]-\frac{\hbar^{2}}{2 \mu_{3 A}} \frac{\partial^{2}}{\partial r_{3 A}^{2}}+\frac{\left(\mathbf{j}_{A}-\mathbf{L}_{12}\right)^{2}}{2 \mu_{3 A} r_{3 A}^{2}}
$$

where $\mu_{i A}$ is the reduced mass of two points of mass. The $\mathbf{j}_{A}$ is the total angular momentum operator of the $\mathrm{CH}_{3}$. The $\mathbf{L}_{12}=\mathbf{L}_{1 A}+\mathbf{L}_{2 A}$ is the total angular momentum operator of the hydrogen atoms $\left(\mathrm{H}_{1} \mathrm{H}_{2} \mathrm{H}_{3}\right)$ in the $\mathrm{CH}_{3}$. The rovibrational kinetic operator of $\mathrm{OH}, H_{r v}^{(B)}$, is written as

$$
H_{r v}^{(B)}=-\frac{\hbar^{2}}{2 \mu_{B}} \frac{\partial^{2}}{\partial r_{B}^{2}}+\frac{j_{B}^{2}}{2 \mu_{B} r_{B}^{2}},
$$

where $\mu_{B}$ is the reduced mass of the diatomic $\mathrm{OH}$ and the $\mathbf{j}_{B}$ is the angular momentum operator of the $\mathrm{OH}$.

The inter-monomer interaction Hamiltonian, $H_{\text {int }}$, is given by

$$
H_{\text {int }}=-\frac{\hbar^{2}}{2 \mu} \frac{\partial^{2}}{\partial R^{2}}+\frac{\left(\mathbf{J}-\mathbf{j}_{A B}\right)^{2}}{2 \mu R^{2}}+V,
$$


where $\mu$ represents the reduced mass of the two monomers in methanol and the $\mathbf{j}_{\mathbf{A B}}=\mathbf{j}_{\mathbf{A}}+\mathbf{j}_{\mathbf{B}}$ is the coupled internally rotational angular momentum. The potential $V$ in Eq. (2.6) is a function of twelve internally vibrational coordinates. The expression of the Hamiltonian operator shows that the Jacobi coordinates provide the exact kinetic operator and allow one to select a physical axis of quantization. Especially, the matrix elements of the Hamiltonian derived above can be conveniently evaluated in an adequate representation. It is worth to note that the MS group ${ }^{11}$ that the molecule under consideration belongs to can be used to extremely simplify the evaluation of the matrix elements and help one classify the energy levels.

In the present work, we only discuss the case of the CO-stretch-torsionrotation. That means all of the vibrational modes are frozen except the COstretching and torsional modes. Thus, the Hamiltonian given in Eq. (2. 3) becomes

$$
\begin{aligned}
H & =-\frac{\hbar^{2}}{2 I_{A}} \frac{\partial^{2}}{\partial \phi_{A}^{2}}-\frac{\hbar^{2}}{2 I_{B}} \frac{\partial^{2}}{\partial \phi_{B}^{2}}-\frac{\hbar^{2}}{2 \mu} \frac{\partial^{2}}{\partial R^{2}}+\frac{\left(\mathbf{J}-\mathbf{j}_{A B}\right)^{2}}{2 \mu R^{2}}+V \\
& =-\frac{\hbar^{2}}{2 \mu} \frac{\partial^{2}}{\partial R^{2}}+H_{\text {ang }}(R),
\end{aligned}
$$

where $I_{A}$ and $I_{B}$ are the inertias of $\mathrm{CH}_{3}$ and $\mathrm{OH}$ around the $z$ axis, respectively.

To construct the matrix of the Hamiltonian in Eq. (2. 7), the total spectral representation is written as the direct product $\Re_{\text {ang }} \otimes \Re_{\text {rad }}$ with

$$
\Re_{\text {rad }}=\left\{\left|\psi_{v_{c o}}\right\rangle, v_{c o}=1, N_{R}\right\},
$$

where $\psi_{v_{c o}}$ is the basis function in the radial degree of freedom of $R$. The angular basis $\Re_{\text {ang }}$ is taken as

$$
\Re_{\text {ang }}=\left\{\left|k_{A}, k_{B}\right\rangle\right\} \otimes\{|J, M, K\rangle\},
$$

where the Wigner functions $\{|j, k, m\rangle\}$ are defined as

$$
\langle\phi, \theta, \chi \mid j, k, m\rangle=\sqrt{\frac{2 j+1}{8 \pi^{2}}} D_{m k}^{j *}(\phi, \theta, \chi)
$$

with the Wigner rotation $D$-matrices defined by

$$
D_{m k}^{j}(\phi, \theta, \chi)=e^{-i m \phi} d_{m k}^{j}(\cos \theta) e^{-i k \chi} .
$$

By introducing $\gamma=\phi_{B}-\phi_{A}$ and $\Phi=\phi_{B}+\phi_{A}$, the Hamiltonian in Eq. (2. 7) and the corresponding basis vector become

$$
H=-\frac{\hbar^{2}}{2 I_{+}}\left(\frac{\partial^{2}}{\partial \gamma^{2}}+\frac{\partial^{2}}{\partial \Phi^{2}}\right)-\frac{\hbar^{2}}{I_{-}} \frac{\partial^{2}}{\partial \gamma \partial \Phi}-\frac{\hbar^{2}}{2 \mu} \frac{\partial^{2}}{\partial R^{2}}+\frac{\left(\mathbf{J}-\mathbf{j}_{A B}\right)^{2}}{2 \mu R^{2}}+V,
$$

and

$$
\left\langle\phi_{A}, \phi_{B} \mid k_{A}, k_{B}\right\rangle=\frac{1}{2 \pi} e^{i\left(k_{A} \phi_{A}+k_{B} \phi_{B}\right)}=\langle\gamma, \Phi \mid m, K\rangle=\frac{1}{2 \pi} e^{1 / 2 i(K \Phi+m \gamma)},
$$


where $I_{ \pm}=I_{A} I_{B} /\left(I_{A} \pm I_{B}\right), m=k_{B}-k_{A}$, and $K=k_{A}+k_{B}$. The matrix elements of the term, $-1 / 2 \mu \partial^{2} / \partial R^{2}$, are of the analytical form in sinc basis function. In our calculation, the $R$ dependence of the basis is given by a discrete variable representation (DVR). ${ }^{12-14}$ Thus, the matrix representation of the the Hamiltonian is sparse. The energy levels are obtained by diagonalizing the Hamiltonian in Eq. (2. 12).

\section{$3 \quad$ Results and Discussions}

First, the model Hamiltonian given in Sec. 2. 1 is used to analyze all of published spectrum data listed in Ref. 2. The coordinate dependent terms are grouped to provide an effective potential,

$$
\begin{aligned}
V= & \frac{1}{2} f_{2} z^{2}+\frac{V_{3}}{2}(1-\cos 3 \gamma)+\frac{V_{6}}{2}(1-\cos 6 \gamma) \\
& +z^{2}\left[{ }^{2} V_{3}(1-\cos 3 \gamma)+{ }^{2} V_{6}(1-\cos 6 \gamma)\right],
\end{aligned}
$$

which describes an essential part of the torsional problem if multiple CO-stretching states are to be treated simultaneously. The values of the parameters in Eq. (3. 1) are listed in Table 1. The data set that is used in the present fitting consists of 295 microwave (MW) and millimeter wave (MMW) transitions with $v_{c o}=0, v_{t} \leq 2$ and $J \leq 5,737$ Fourier transform far-infrared (FIR) data involving energy levels with $v_{c o} \leq 1, v_{t} \leq 4$ and $J \leq 5$ for all available $K$ states of $A$ and $E$ torsional symmetries. ${ }^{2}$ All of the MW and MMW lines have experimental uncertainties of $100 \mathrm{kHz}$ and the experimental uncertainties of the Fourier transform FIR data are $0.0003 \mathrm{~cm}^{-1}=9 \mathrm{MHz}$. To reflect the difference between the experimental uncertainties of the MW, MMW and FIR transitions, all of MW and MMW transitions were given a weight that is 100 times larger than that for the FIR data. Using 25 adjustable and 8 fixed parameters the fit converged with a root-mean-square (rms) deviation of $0.20 \mathrm{~cm}^{-1}$ for 1032 experimental data with $v_{c o} \leq 1, v_{t} \leq 4, J \leq 5$, and $|K| \leq 5$. The $295 \mathrm{MW}$ and MMW transitions were fit with a rms deviation of $2.1 \mathrm{MHz}$ while 737 FIR data had a rms deviation of $0.238 \mathrm{~cm}^{-1}$. The present results show that the relative location of the vibrational ground state and the $\mathrm{CO}$ stretch fundamental state can be well reproduced for torsional states with $v_{t} \leq 4, J \leq 5$, and $|K| \leq 5$ using the simple form of the effective potential in Eq. (3. 1). The first two columns of Table 1 provide the parameters and corresponding operators in the reduced torsion-rotational Hamiltonian for $\mathrm{CH}_{3} \mathrm{OH}$ as well as the parameters and operators that were included in previous studies of this system. The values of the parameters that were obtained in the present fit are listed in the third column and their uncertainties $(1 \sigma)$ are given in parentheses. The fourth column provides the results of fit to the transitions with $v_{c o}=1$ by Henningsen. ${ }^{15}$ The fifth column provides the constants fitted by Duan et al,,$^{9}$ where $470 \mathrm{MW}$ and MMW transitions with $v_{c o}=0$ were fit with a rms deviations of $0.35 \mathrm{MHz}$ using 28 terms. 
Table 1: Molecular constants used for representing the CO-stretch-torsionrotation of $\mathrm{CH}_{3} \mathrm{OH}$

\begin{tabular}{|c|c|c|c|c|}
\hline Operator & Constant ${ }^{a}$ & $\begin{array}{c}\text { Current fit } \\
\mathrm{v}_{c o}=0,1\end{array}$ & $\begin{array}{c}\mathrm{Fit}^{\mathrm{c}} \\
\mathrm{v}_{\mathrm{co}}=1\end{array}$ & $\begin{array}{c}\text { Fit }^{d} \\
v_{c o}=0\end{array}$ \\
\hline$P_{a}^{2}$ & $A$ & $127520.580(4006)$ & 127438.32 & $127536.083(6716)$ \\
\hline$P_{b}^{2}$ & $B$ & $24685.496(1149)$ & 24479.01 & $24687.6247(336)$ \\
\hline$P_{c}^{2}$ & $C$ & $23764.049(1077)$ & 23548.38 & $23762.4232(1040)$ \\
\hline$\left\{\stackrel{c}{P}_{a}, P_{b}\right\}$ & $D_{a b}$ & $-151.3(33)$ & & $-114.496(1590)$ \\
\hline$P_{\gamma}^{2}$ & $F\left(\mathrm{~cm}^{-1}\right)$ & $27.737805(4606)$ & 27.608226 & $27.6447930(8785)$ \\
\hline & $\rho$ (unitless) & $0.810211(7)$ & & 0.809883 \\
\hline$-P^{4}$ & $\triangle_{J}$ & 0.0518 (fixed) & 0.049 & $0.05021(6)$ \\
\hline$-P^{2} P_{a}^{2}$ & $\triangle_{J K}$ & $0.2793(947)$ & 0.286 & $0.2793(982)$ \\
\hline$-P_{a}^{4} a$ & $\Delta_{K}$ & $1.3053(1570)$ & 1.14 & $1.6106(266)$ \\
\hline$-2 P^{2}\left(P_{b}^{2}-P_{c}^{2}\right)$ & $\delta_{J}$ & 0.0018 (fixed) & & $0.00169(7)$ \\
\hline$P^{2} P_{\gamma}^{2}$ & $a_{V}$ & $-3.5046(1293)$ & -5.01 & $-3.5506(26)$ \\
\hline$P^{2}(1-\cos 3 \gamma)$ & $F_{V}$ & $-71.650(1219)$ & -196.2 & $-71.49904(3)$ \\
\hline$P^{2} P_{a} P_{\gamma}$ & $L_{V}$ & 0.0942 (fixed) & -0.068 & $0.0809(11)$ \\
\hline$P_{g}^{3} P_{\gamma}$ & $k_{1}$ & $-3.116(150)$ & -1.44 & $-1.6339(1070)$ \\
\hline$P_{a}^{2} P_{\gamma}^{2}$ & $k_{2}$ & $-63.833(1593)$ & -55.2 & $-66.445(1115)$ \\
\hline$P_{a} P_{\gamma}^{3}$ & $k_{3}$ & $-178.290(1519)$ & -161.1 & $-122.009(2275)$ \\
\hline$P_{\gamma}^{4}$ & $k_{4}$ & $-259.102(1302)$ & -256.3 & $-218.7($ fixed) \\
\hline$P_{a}^{2}(1-\cos 3 \gamma)$ & $k_{5}$ & $343.33(1266)$ & 410.9 & $325.93(1071)$ \\
\hline$\left\{P_{a}, P_{c}\right\} \sin 3 \gamma$ & $k_{7}^{\prime}$ & $-328.522(1250)$ & & $-274.52(1529)$ \\
\hline$\left\{P_{\gamma}^{2}, P_{b}^{2}-P_{c}^{2}\right\}$ & $c_{1}$ & $1.6($ fixed $)$ & -1.2272 & $-1.6632(79)$ \\
\hline$\left\{P_{\gamma}^{2},\left\{P_{a}, P_{b}\right\}\right\}$ & $\triangle_{a b}$ & $-2.891(1362)$ & & $-6.9643(6738)$ \\
\hline$\left\{P_{a}, P_{b}\right\}(1-\cos 3 \gamma)$ & $d_{a b}$ & $333.56(3341)$ & & $304.800(2591)$ \\
\hline$P_{a} P_{\gamma}^{5}$ & $K_{7}$ & $0.7658(889)$ & & \\
\hline$P_{a}^{2} P_{\gamma}^{4}$ & $K_{1}$ & 0.2327 (fixed) & & \\
\hline$P_{a}^{3} P_{\gamma}^{3}$ & $K_{3}$ & $-0.2663(1703)$ & & \\
\hline$\left\{P_{\gamma} P_{a},\left\{P_{a}^{2}, P_{b}^{2}-P_{c}^{2}\right\}\right\}$ & $c_{10}$ & 0.2183 (fixed) & & \\
\hline$\left\{P_{b}^{2}-P_{c}^{2}, P_{a} P_{\gamma}^{3}\right\}$ & $c_{12}$ & 0.2109 (fixed) & & \\
\hline$\left(P_{b}^{2}-P_{c}^{2}\right)\left\{P_{\gamma}^{2}, 1-\cos 3 \gamma\right\}$ & $c_{19}$ & 0.2419 (fixed) & & \\
\hline$(1-\cos 3 \gamma) / 2$ & $V_{3}\left(\mathrm{~cm}^{-1}\right)$ & $369.45509(8690)$ & 392.35 & $373.37459(3352)$ \\
\hline$(1-\cos 6 \gamma) / 2$ & $V_{6}\left(\mathrm{~cm}^{-1}\right)$ & $6.3653(1379)$ & -0.52 & $-0.93196(4757)$ \\
\hline$z^{2}$ & $\mathrm{f}_{2}(\mathrm{SI})^{b}$ & $507.6181(864)$ & & \\
\hline$z^{2}(1-\cos 3 \gamma)$ & ${ }^{2} \mathrm{~V}_{3}(\mathrm{SI})^{b}$ & $1.9856(371)$ & & \\
\hline$z^{2}(1-\cos 6 \gamma)$ & ${ }^{2} \mathrm{~V}_{6}(\mathrm{SI})^{b}$ & $-3.7463(561)$ & & \\
\hline$z^{2} P_{\gamma}^{2}$ & ${ }^{2} \mathrm{~F} \times 10^{-33}(\mathrm{SI})^{b}$ & $5.381(25)$ & & \\
\hline
\end{tabular}

$a$
$b$ Parameters in $\mathrm{MHz}$.

$b$ Vibrational and related parameters in SI.

$c$ Ref. 15.
$e$ Ref. 9.

Using the fitted PES in Eq. (3. 1), we also calculated the CO-stretchingrotational energy levels based on the quantum mechanical Hamiltonian. Table 2 gives the comparison of the two kinds of calculated energy levels with experimental results for $J=2$, where a total of 50 levels are arranged in order of frequency alone with the observed VTR energy levels. The table contains quantum numbers for the states, residuals (observed-fitted levels and observedcalculated levels), the symmetry ( $A$ or $E$ ) of the torsional sub-states, and the parity quantum number $(+,-)$ that refer only to $A$ torsional sub-states. The present results shown that the relative location of the vibrational ground state and the $\mathrm{CO}$ stretch fundamental state are well reproduced for torsional states with $v_{t} \leq 2$ using the quantum mechanical simulation. However, the rms of the deviation of the variational values with the experimental ones is about 2.2 $\mathrm{cm}^{-1}$ that is about 10 times larger than that in model Hamiltonian. The reason may result either from the approximated model or form the inaccurate potential 
Table 2: Energy levels of $\mathrm{CH}_{3} \mathrm{OH}$ in unit of $\mathrm{cm}^{-1 a}$

\begin{tabular}{|c|c|c|c|c|c|c|c|c|c|c|}
\hline$\overline{\mathrm{A} / \mathrm{E}}$ & $\overline{v_{c o}}$ & $\overline{v_{t}}$ & $\overline{\bar{J}}$ & $\overline{K K}$ & $P$ & $\overline{\operatorname{Exp}} .^{b}$ & $\overline{\mathrm{Fit}^{c}}$ & E-F ${ }^{d}$ & $\mathrm{Cal}^{e}$ & $\overline{\text { E-C }}{ }^{f}$ \\
\hline & & & & & & & $\mathrm{J}=2$ & & & \\
\hline A & 0 & 0 & 2 & 0 & + & 4.84049 & 4.84047 & .000 & 5.51544 & -.675 \\
\hline $\mathrm{E}$ & 0 & 0 & 2 & -1 & & -.40543 & -.40529 & .000 & -.00014 & -.405 \\
\hline $\mathrm{E}$ & 0 & 0 & 2 & 0 & & 4.84073 & 4.84073 & .000 & 5.51533 & -.675 \\
\hline A & 0 & 0 & 2 & 1 & + & 14.90430 & 14.90422 & .000 & 15.45369 & $-.54 \mathrm{~s}$ \\
\hline A & 0 & 0 & 2 & 1 & - & 14.98778 & 14.98770 & .000 & 15.47501 & -.487 \\
\hline $\mathrm{E}$ & 0 & 0 & 2 & 1 & & 10.34657 & 10.34662 & .000 & 10.46546 & -.119 \\
\hline $\mathrm{E}$ & 0 & 0 & 2 & 2 & & 11.17828 & 11.17843 & .000 & 10.64262 & .536 \\
\hline $\mathrm{E}$ & 0 & 0 & 2 & -2 & & 13.71898 & 13.71917 & .000 & 13.46315 & .256 \\
\hline A & 0 & 0 & 2 & 2 & - & 31.04901 & 31.04893 & .000 & 30.42347 & .626 \\
\hline A & 0 & 0 & 2 & 2 & + & 31.04905 & 31.04900 & .000 & 30.44477 & .604 \\
\hline $\mathrm{E}$ & 0 & 1 & 2 & 1 & & 198.29105 & 198.27993 & .011 & 199.22743 & -.936 \\
\hline A & 0 & 1 & 2 & 2 & + & 212.41775 & 212.40593 & .012 & 212.34853 & .069 \\
\hline A & 0 & 1 & 2 & 2 & - & 212.41775 & 212.44905 & -.031 & 212.34853 & .068 \\
\hline $\mathrm{E}$ & 0 & 1 & 2 & 0 & & 204.61844 & 204.60729 & .011 & 205.43830 & -.820 \\
\hline A & 0 & 1 & 2 & 1 & + & 230.88932 & 230.87744 & .012 & 230.53584 & .353 \\
\hline A & 0 & 1 & 2 & 1 & & 230.89894 & 230.88712 & .012 & 230.55709 & .342 \\
\hline $\mathrm{E}$ & 0 & 1 & 2 & -2 & & 249.12439 & 249.11268 & .012 & 246.67994 & 2.444 \\
\hline $\mathrm{E}$ & 0 & 1 & 2 & 2 & & 273.64737 & 273.63810 & .009 & 276.04858 & -2.401 \\
\hline $\mathrm{E}$ & 0 & 1 & 2 & -1 & & 283.01760 & 283.00952 & .008 & 285.05823 & -2.041 \\
\hline A & 0 & 1 & 2 & 0 & + & 299.27974 & 299.27187 & .008 & 300.65626 & -1.377 \\
\hline A & 0 & 2 & 2 & 0 & + & 358.04034 & 358.04295 & -.003 & 359.30377 & -1.263 \\
\hline $\mathrm{E}$ & 0 & 2 & 2 & -1 & & 365.47979 & 365.48121 & -.001 & 365.21149 & .268 \\
\hline $\mathrm{E}$ & 0 & 2 & 2 & 2 & & 403.15497 & 403.15451 & .000 & 399.82158 & 3.333 \\
\hline $\mathrm{E}$ & 0 & 2 & 2 & -2 & & 443.95855 & 443.97235 & -.014 & 449.40802 & -5.449 \\
\hline A & 0 & 2 & 2 & 1 & + & 479.37273 & 479.39099 & -.018 & 483.96187 & -4.589 \\
\hline A & 0 & 2 & 2 & 1 & & 479.39093 & 479.40920 & -.018 & 483.98276 & -4.592 \\
\hline $\mathrm{E}$ & 0 & 2 & 2 & 0 & & 506.01909 & 506.03283 & -.014 & 508.81335 & -2.794 \\
\hline $\mathrm{E}$ & 0 & 2 & 2 & 1 & & 551.00435 & 551.00722 & -.003 & 551.13618 & -.132 \\
\hline A & 0 & 2 & 2 & 2 & - & 614.14008 & 614.13037 & .010 & 610.81209 & 3.328 \\
\hline A & 0 & 2 & 2 & 2 & + & 614.14008 & 614.13037 & .010 & 610.81209 & 3.328 \\
\hline A & 1 & 0 & 2 & 0 & + & 1038.68345 & 1038.77356 & -.090 & 1040.03541 & -1.352 \\
\hline $\mathrm{E}$ & 1 & 0 & 2 & -1 & & 1033.91137 & 1033.51629 & .395 & 1034.43474 & -.523 \\
\hline $\mathrm{E}$ & 1 & 0 & 2 & 0 & & 1038.49648 & 1038.50936 & -.013 & 1039.91482 & -1.418 \\
\hline A & 1 & 0 & 2 & 1 & + & 1048.75741 & 1048.64912 & .108 & 1049.88171 & -1.124 \\
\hline A & 1 & 0 & 2 & 1 & & 1048.84413 & 1048.73253 & .112 & 1050.31464 & -1.471 \\
\hline $\mathrm{E}$ & 1 & 0 & 2 & 1 & & 1043.83049 & 1043.94868 & -.118 & 1044.98585 & -1.155 \\
\hline $\mathrm{E}$ & 1 & 0 & 2 & 2 & & 1045.42780 & 1045.06618 & .362 & 1045.41899 & .009 \\
\hline $\mathrm{E}$ & 1 & 0 & 2 & -2 & & 1047.77162 & 1047.53605 & .236 & 1047.88661 & -.115 \\
\hline A & 1 & 0 & 2 & 2 & + & 1064.43044 & 1064.61015 & -.180 & 1063.38838 & 1.042 \\
\hline A & 1 & 0 & 2 & 2 & - & 1064.43044 & 1064.61015 & -.180 & 1063.38838 & 1.042 \\
\hline $\mathrm{E}$ & 1 & 1 & 2 & 1 & & 1232.62536 & 1232.38543 & .240 & 1234.32138 & -1.696 \\
\hline A & 1 & 1 & 2 & 2 & - & 1246.87386 & 1246.63343 & .240 & 1247.47400 & -.600 \\
\hline A & 1 & 1 & 2 & 2 & + & 1246.87386 & 1246.63343 & .240 & 1247.47400 & -.600 \\
\hline $\mathrm{E}$ & 1 & 1 & 2 & 0 & & 1238.68106 & 1238.48414 & .197 & 1240.41529 & -1.734 \\
\hline A & 1 & 1 & 2 & 1 & + & 1264.38316 & 1264.41266 & $\begin{array}{l}. .029 \\
-.029\end{array}$ & 1265.32102 & -.938 \\
\hline A & 1 & 1 & 2 & 1 & - & 1264.39137 & 1264.42377 & -.032 & 1265.75318 & -1.362 \\
\hline $\mathrm{E}$ & 1 & 1 & 2 & -2 & & 1281.81559 & 1282.16204 & -.346 & 1281.57984 & .236 \\
\hline $\mathrm{E}$ & 1 & 1 & 2 & -1 & & $* 1305.26255$ & 1313.74774 & -8.485 & 1317.59291 & -12.330 \\
\hline $\mathrm{E}$ & 1 & 1 & 2 & 2 & & & 1305.75609 & & 1309.68118 & \\
\hline A & 1 & 1 & 2 & 0 & + & & 1328.70054 & & 1332.13530 & \\
\hline
\end{tabular}

a Energy levels are arranged from lower to higher and are relative to the lowest one for $A$ and $E$, respectively.

$b$ Experimental results are taken from Ref. 2. The levels marked with' $*$ ' are not included in the fitted data set. $c$ The present fit results based on a model Hamiltonian.

$d \mathrm{E}-\mathrm{F}=$ the experimental-the fitted values.

$e$ The present calculated results based on the variational calculation.

$f \mathrm{E}-\mathrm{C}=$ the experimental-the variational values.

function, which is being investigated.

In conclusion, we have used two kinds of the 5D Hamiltonian models, i.e., perturbation method and perturbation-free method, to investigate the CO-stretchingtorsion-rotational states. The model Hamiltonian is used to analyze the observed data that involve the torsional and CO-stretching modes for $\mathrm{CH}_{3} \mathrm{OH}$ to generate global values for the molecular parameters. The relative location of the vibrational ground state and the CO stretch fundamental state is well reproduced for torsional states with $v_{t} \leq 4$ and $J \leq 5$. We have proposed a Hamiltonian for calculating VTR energy levels of methanol through using the Jacobi vectors. The present results for the $\mathrm{CO}$-stretching-torsion-rotation states shown that present 
formulation can be used to carry out a multi-dimensional quantum calculation for methanol or to fit the potential energy surface. On balance, the presented methods of analysis provide an important first step in an attempt to model various small amplitude vibrational interactions influenced by the internal rotation motion and to interpret observed spectrum data.

\section{ACKNOWLEDGMENTS}

The authors gratefully acknowledge to support from Office of Research at The Ohio State University and the Petroleum Research Fund administered by the American Chemical Society.

\section{References}

[1] C. C. Lin and J. D. Swalen, Rev. Mod. Phys. 31 (1959) 841-892.

[2] G. Moruzzi, B. P. Winnewisser, M. Winnewisser, I. Mukhopadhyay, and F. Strumia, Microwave, Infrared and Laser Transitions of Methanol, CRC Press, (1995).

[3] Y. B. Duan and K. Takagi, Phys. Lett. A 207 (1995) 203-208.

[4] Y. B. Duan, H. M. Zhang, and K. Takagi, J. Chem. Phys., 104 (1996) 3914-3922.

[5] Y. B. Duan, L. Wang, I. Mukhopadhyay, and K. Takagi, J. Chem. Phys., 110 (1999) 927-935.

[6] Y. B. Duan, L. Wang, and K. Takagi, J. Mol. Spectrosc. 193 (1999) 418-433.

[7] Y. B. Duan and A. B. McCoy, J. Mol. Spectrosc., 199 (2000) 302-306.

[8] Y. B. Duan, L. Wang, X. T. Wu, I. Mukhopadhyay, and K. Takagi J. Chem. Phys., 111 (1999) 2385-2391.

[9] Y. B. Duan, A. B. McCoy, L. Wang, and K. Takagi, J. Chem. Phys., 112 (2000) 212-219.

[10] L. Wang, Y. B. Duan, I. Mukhopadhyay, D. S. Perry, and K. Takagi, Chem. Phys., 263 (2001) 263-270.

[11] P. R. Bunker and P. Jensen, Molecualr Symmetry and Spectroscopy, NRC Research Press (1998), Ottawa.

[12] J. V. L. Lill, G. A. Parker, J. C. Light, Chem. Phys. Lett. 89 (1982) 483-489.

[13] J. C. Light and Z. Bacic, J. Chem. Phys. 87 (1987) 4008-4019.

[14] D. T. Colbert and W. H. Miller, J. Chem. Phys. 96 (1992) 1982-1992.

[15] J. O. Henningsen, J. Mol. Spectrosc. 85 (1981) 282-300. 